\title{
Spin statistics for triplet-triplet annihilation upconversion: exchange coupling, intermolecular orientation and reverse intersystem crossing
}

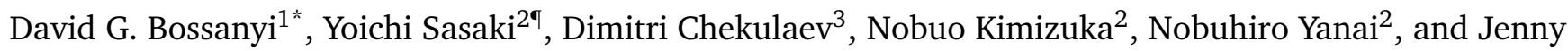 \\ Clark $^{1 *}$
}

${ }^{1}$ Department of Physics and Astronomy, The University of Sheffield, Sheffield, UK

${ }^{2}$ Department of Chemistry and Biochemistry, Graduate School of Engineering, Kyushu University, Fukuoka, Japan

${ }^{3}$ Department of Chemistry, The University of Sheffield, Sheffield, UK

'Present address: University of Strasbourg, CNRS, ISIS, 8 allée G. Monge, 67000, Strasbourg, France

*E-mail: dgbossanyi1@sheffield.ac.uk, jenny.clark@sheffield.ac.uk

\begin{abstract}
Triplet-triplet annihilation upconversion (TTA-UC) has great potential for significantly improving the light harvesting capabilities of photovoltaic cells, as well as being sought after for biomedical applications. Many factors combine to influence the overall efficiency of TTA-UC, the most fundamental of which is the spin statistical factor, $\eta$, that gives the probability that a bright singlet state is formed from a pair of annihilating triplet states. Using solid rubrene as a model system, we reiterate why experimentally measured magnetic field effects prove that annihilating triplets first form weakly exchange-coupled triplet-pair states. This is contrary to conventional discussions of TTA-UC that implicitly assume strong exchange coupling and we show that it has profound implications for the spin statistical factor $\eta$. For example, variations in intermolecular orientation tune $\eta$ from $\frac{2}{5}$ to $\frac{2}{3}$ through spin mixing of the tripletpair wavefunctions. Since the fate of spin-1 triplet-pair states is particularly crucial in determining $\eta$, we investigate it in rubrene using pump-push-probe spectroscopy and find additional evidence for the recently reported high-level reverse intersystem crossing channel. We incorporate all of these factors into an updated model framework in which to understand the spin statistics of TTA-UC, and use it to rationalise the differences in reported values of $\eta$ amongst different common annihilator systems. We suggest that harnessing high-level reverse intersystem crossing channels in new annihilator molecules may be a highly promising strategy to exceed any spin statistical limit.
\end{abstract}

\section{Introduction}

Photon upconversion, whereby low energy photons are combined to produce light at a shorter wavelength is highly sought after as a strategy for boosting the efficiency of conventional photovoltaics ${ }^{1,2}$, as well as for biomedical applications ${ }^{3,4}$ including targeted drug delivery ${ }^{5}$ and optogenetics ${ }^{6}$. To be technologically useful, the upconversion process should be efficient in solid materials under weak, incoherent illumination ${ }^{7}$.

Triplet-triplet annihilation upconversion (TTA-UC) offers the potential to fulfil these criteria $^{7-10}$. During TTAUC, photo-excitation of a donor species results in the sensitization of triplet states on acceptor molecules. Subsequent annihilation of two triplets yields an excited singlet state of approximately twice the triplet energy which can fluoresce, giving upconverted emission ${ }^{11}$.

The overall quantum efficiency $\Phi_{U C}$ of TTA-UC can be written as the product of the yields of the constituent steps $^{12}$ :

$$
\Phi_{U C}=\frac{1}{2} \eta \Phi_{P L} \Phi_{T T A} \Phi_{T E T} \Phi_{I S C} .
$$

In this expression, $\Phi_{I S C}, \Phi_{T E T}$ and $\Phi_{P L}$ are the quantum yields of intersystem crossing (or more generally triplet production) on the donor, triplet energy transfer from donor to acceptor and acceptor fluorescence respectively. The remaining terms combine to give the quantum yield of triplet-triplet annihilation. The factor of $\frac{1}{2}$ reflects the fact that two triplets yield one singlet and $\Phi_{T T A}$ describes the competition between annihilation and decay for the fate of triplet states. The spin statistical factor $\eta$ gives the likelihood of obtaining a spin-0 singlet state from the annihilation of two spin-1 triplets. 
TTA-UC systems with near unity $\Phi_{I S C}{ }^{6,13,14}, \Phi_{T E T}{ }^{6,15}$ and $\Phi_{P L}{ }^{14-17}$ have been demonstrated and whilst maximising $\Phi_{T T A}$ at low light intensities remains a challenge in the solid state ${ }^{7}$, there is no intrinsic reason why this cannot be achieved. In principal therefore, $\eta$ is the only factor that could impose a fundamental limit on the efficiency of TTA-UC.

The spin statistical factor for TTA-UC is almost always discussed in terms of nine pure-spin triplet-pair encounter complexes: one spin-0 singlet, three spin- 1 triplets and five spin-2 quintets ${ }^{12,14,17-20}$. At first glance, this might suggest that $\eta=\frac{1}{9}$, however measurements of $\Phi_{U C}$ greatly exceeding this limit demonstrate that this is not the case ${ }^{18,21}$. The quintet complexes readily dissociate again into individual triplets since molecular quintet states are energetically inaccessible in relevant molecules ${ }^{22}$. The triplet complexes on the other hand can undergo internal conversion to nearby triplet states, leading to the loss of one triplet of the pair ${ }^{19,20}$. If such internal conversion is efficient, this description yields $\eta=\frac{2}{5}$.

These conventional discussions of spin statistics overlook many of the subtleties of triplet-triplet interactions, studied initially by Merrifield and co-workers 50 years ago ${ }^{23}$. Such interactions have been investigated in great depth more recently through research into the reverse process to triplet-triplet annihilation: singlet fission ${ }^{24,25}$, whereby pairs of triplet excitons are produced from singlets via the same intermediate triplet-pair states ${ }^{26}$. Here we aim to bridge the apparent divide between the singlet fission and TTA-UC descriptions by demonstrating the profound effect of triplet-pair character, in particular the strength of inter-triplet exchange coupling, on the spin statistical factor. Inspired by recent reports of high-level reverse intersystem crossing from $\mathrm{T}_{2}$ to $\mathrm{S}_{1}{ }^{27}$, which could allow the loss associated with the formation of triplet complexes to be bypassed ${ }^{28}$, we also investigate internal conversion rates and the fate of higher-lying triplet states and their impacts on the spin statistical factor.

We therefore begin by providing an overview of the spin physics of triplet-pair states in the context of TTA-UC. Next, we investigate the triplet-pair character, energy levels, internal conversion rates and reverse intersystem crossing in rubrene, the most common acceptor molecule for near-infrared-to-visible TTA-UC ${ }^{9,17}$. Based on these experimental results, we present an updated model for the spin statistics of upconversion that includes the effects of inter-triplet exchange coupling and orientation, as well as internal conversion rates, energy levels and reverse intersystem crossing. We find that variations in exchange energy and orientation can tune the spin statistical factor $\eta$ within the range $\frac{2}{5} \leq \eta \leq \frac{2}{3}$, but that careful optimisation of the $\mathrm{S}_{1}, \mathrm{~T}_{2}$ and $\mathrm{T}_{1}$ energy levels may allow $\eta$ to reach unity, thereby bypassing such considerations.

\section{Theoretical background}

Recent reviews have discussed the current understanding of the spin physics of triplet-pair states in great depth ${ }^{24,25}$. Here, we review the important points and relate them to the spin statistical factor $\eta$.

Individual triplet states are governed by a spin Hamiltonian comprising (in the absence of spin-orbit coupling and other perturbations) a Zeeman term describing the effect of external magnetic fields $\boldsymbol{B}$ and an intra-triplet dipoledipole coupling term, parametrised by the so-called zero-field splitting parameters $D$ and $E$ :

$$
\begin{aligned}
\hat{H} & =\hat{H}_{Z \text { eeman }}+\hat{H}_{z \text { ero-field }} \\
& =g \mu_{B} \boldsymbol{B} \cdot \hat{\boldsymbol{S}}+D\left(\hat{S}_{z}^{2}-\frac{1}{3} \hat{\boldsymbol{S}}^{2}\right)+E\left(\hat{S}_{x}^{2}-\hat{S}_{y}^{2}\right),
\end{aligned}
$$

where $\hat{\boldsymbol{S}}$ is the 2-electron spin operator. In the $\boldsymbol{B}=0$ limit, the three triplet eigenstates are given by

$$
\begin{aligned}
& |x\rangle=\frac{1}{\sqrt{2}}(|\downarrow \downarrow\rangle-|\uparrow \uparrow\rangle) \\
& |y\rangle=\frac{i}{\sqrt{2}}(|\downarrow \downarrow\rangle+|\uparrow \uparrow\rangle) \\
& |z\rangle=\frac{1}{\sqrt{2}}(|\uparrow \downarrow\rangle+|\downarrow \uparrow\rangle),
\end{aligned}
$$

where the arrows indicate the individual electron spin states. Since we use rubrene as our model system, we define our coordinate system such that $x$ is parallel to the long molecular axis, $y$ is parallel to the short axis and $z$ is perpendicular 
to the tetracene backbone plane ${ }^{29}$.

The spin Hamiltonian for a pair of interacting triplet states, labelled $A$ and $B$, can be written as

$$
\hat{H}=\hat{H}_{\text {exchange }}+\hat{H}_{\text {inter }}+\sum_{i=A, B}\left(\hat{H}_{\text {Zeeman }, i}+\hat{H}_{\text {zero-field }, i}\right) .
$$

In addition to the 2-electron spin Hamiltonians (equation 2) for individual triplets on molecules $A$ and $B$, there are two additional inter-triplet terms that couple their spins together. First, there is an inter-triplet exchange interaction of strength $J$ which requires wavefunction overlap between the two triplets in the pair. Second is an inter-triplet spin-dipolar coupling term, which is a longer range, through-space interaction. The total spin Hamiltonian becomes ${ }^{25}$

$$
\begin{aligned}
\hat{H} & =J \hat{\boldsymbol{S}}_{A} \cdot \hat{\boldsymbol{S}}_{B}+\hat{\boldsymbol{S}}_{A} \cdot D_{\text {inter }} \cdot \hat{\boldsymbol{S}}_{B} \\
& +\sum_{i=A, B}\left[g \mu_{B} \boldsymbol{B} \cdot \hat{\boldsymbol{S}}_{i}+D\left(\hat{S}_{i, z}^{2}-\frac{1}{3} \hat{\boldsymbol{S}}_{i}^{2}\right)+E\left(\hat{S}_{i, x}^{2}-\hat{S}_{i, y}^{2}\right)\right] .
\end{aligned}
$$

The spin-dipolar term can be formulated in various ways. Since the inter-triplet coupling strength, which we label $X$, is thought to be on the order of $10 \mathrm{neV}^{30,31}$, much less than the intra-triplet dipolar coupling $(D \sim 10 \mu \mathrm{eV}, E<D)$ the exact form is unimportant ${ }^{29}$. For simplicity, we take $\hat{\boldsymbol{S}}_{A} \cdot D_{\text {inter }} \cdot \hat{\boldsymbol{S}}_{B} \approx X \hat{\boldsymbol{S}}_{A} \cdot \hat{\boldsymbol{S}}_{B}{ }^{32}$.

A convenient basis set for diagonalising $\hat{H}$ and obtaining the triplet-pair spin wavefunctions $\left|\psi_{l}\right\rangle$ comprises the nine product pair states $|x x\rangle,|x y\rangle, \ldots,|z z\rangle$, where we have dropped the $A, B$ subscripts for clarity. We note that since the $x y z$ coordinate systems of molecules $A$ and $B$ do not in general coincide, a rotation operation must be applied to $\hat{H}_{\text {zero-field,B }}{ }^{29}$. As a result, $\left|\psi_{l}\right\rangle$ carry a dependence on the relative orientation of the two molecules which, as we demonstrate below, has important implications for the spin statistical factor $\eta$.

Recent research has shown the importance of distinguishing between strongly $(J \gg D)$ and weakly $(J \ll D)$ exchange-coupled triplet-pair states ${ }^{33-36}$. In the limit of strong exchange coupling, the eigenstates $\left|\psi_{l}\right\rangle$ of $\hat{H}$ coincide with the nine lowest energy eigenstates of the four-electron spin operator $\left(\hat{\boldsymbol{S}}_{A}+\hat{\boldsymbol{S}}_{B}\right)^{2}$. They are therefore pure spin states (spin is a good quantum number) and comprise one spin- 0 singlet ${ }^{1}$ (TT), three spin- 1 triplets ${ }^{3}$ (TT) and five spin-2 quintets ${ }^{5}(\mathrm{TT})$. In the zero-field basis, the spin wavefunctions can be written as ${ }^{37}$

$$
\begin{gathered}
|S\rangle=\frac{1}{\sqrt{3}}(|x x\rangle+|y y\rangle+|z z\rangle), \\
\left|T_{x}\right\rangle=\frac{1}{\sqrt{2}}(|y z\rangle-|z y\rangle), \\
\left|T_{y}\right\rangle=\frac{1}{\sqrt{2}}(|z x\rangle-|x z\rangle), \\
\left|T_{z}\right\rangle=\frac{1}{\sqrt{2}}(|x y\rangle-|y x\rangle), \\
\left|Q_{a}\right\rangle=\frac{1}{\sqrt{2}}(|x x\rangle-|y y\rangle), \\
\left|Q_{b}\right\rangle=\frac{1}{\sqrt{6}}(|x x\rangle+|y y\rangle-2|z z\rangle), \\
\left|Q_{x}\right\rangle=\frac{1}{\sqrt{2}}(|y z\rangle+|z y\rangle), \\
\left|Q_{y}\right\rangle=\frac{1}{\sqrt{2}}(|z x\rangle+|x z\rangle), \\
\left|Q_{z}\right\rangle=\frac{1}{\sqrt{2}}(|x y\rangle+|y x\rangle) .
\end{gathered}
$$

These are the nine triplet-pair intermediates that are conventionally considered when evaluating the spin statistics of TTA-UC ${ }^{12,14,17-20}$. Thus to date, there has been an implicit assumption that the encounter complexes formed through TTA are strongly exchange-coupled. We explain below why this cannot be the case. 
When $J \ll D$ the eigenstates of $\hat{H}$ are different. In the case of parallel molecules, there are three pure triplets $\left|T_{x, y, z}\right\rangle$ and three pure quintets $\left|Q_{x, y, z}\right\rangle$. The remaining three, $|x x\rangle,|y y\rangle$ and $|z z\rangle$, can be written as mixtures of $|S\rangle$, $\left|Q_{a}\right\rangle$ and $\left|Q_{b}\right\rangle$. In other words, we can no longer consider pure spin states. For non-parallel molecules, additional singlet-triplet and quintet-triplet mixing occurs and all of the eigenstates obtain mixed spin character ${ }^{35}$. We can quantify the character of the eigenstates by calculating their overlap with the appropriate pure spin states. For example the singlet character is given by ${ }^{23,38}$

$$
\left|C_{S}^{l}\right|^{2}=\left|\left\langle S \mid \psi_{l}\right\rangle\right|^{2}
$$

Analogously, we can define the triplet character as ${ }^{39}$

$$
\left|C_{T}^{l}\right|^{2}=\sum_{m=x, y, z}\left|C_{T m}^{l}\right|^{2}=\sum_{m=x, y, z}\left|\left\langle T_{m} \mid \psi_{l}\right\rangle\right|^{2},
$$

and the quintet character as

$$
\left|C_{Q}^{l}\right|^{2}=\sum_{m=a, b, x, y, z}\left|C_{Q m}^{l}\right|^{2}=\sum_{m=a, b, x, y, z}\left|\left\langle Q_{m} \mid \psi_{l}\right\rangle\right|^{2} .
$$

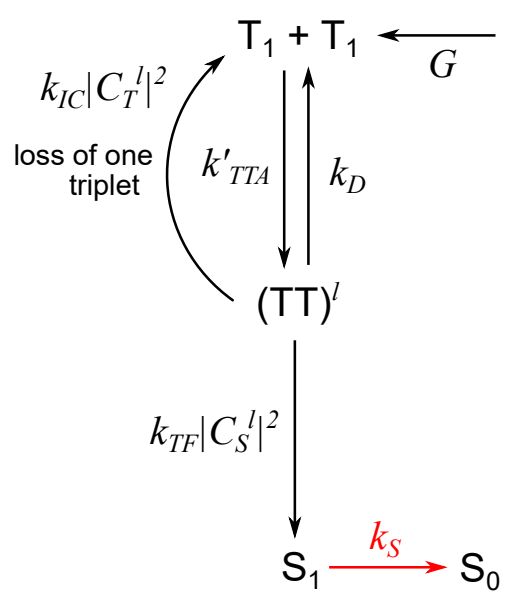

Figure 1. The simplest kinetic model of TTA-UC. A schematic diagram of the simplest kinetic model for TTA-UC that considers triplet-pair spin character in a general way. The processes and rates are described in the text. This is referred to as model 1.

In order to understand the influence of triplet-pair character on the spin statistics of TTA-UC, we can construct a kinetic model based on the Johnson-Merrifield framework ${ }^{23,38}$. We note that while related analyses have been reported by Mezyk et al. in $2009^{39}$ and more recently by Schmidt and Castellano ${ }^{40}$, the effect of triplet-pair character on spin statistics was not explored in either work.

The simplest possible model is illustrated in Fig. 1. Triplet states generated at rate $G$ can annihilate to form triplet-pair states (TT) ${ }^{l}$, whose spin wavefunctions $\left|\psi_{l}\right\rangle$ are determined by equation (5). We choose to consider an annihilation process that depends linearly rather than quadratically on the triplet population. This results in a linear set of rate equations with a simple analytical solution under steady-state conditions. TTA is therefore described by an effective annihilation rate $k_{T T A}^{\prime}$. The final expression for the spin statistical factor (equation (22), below) is identical to that obtained if we instead use a bimolecular, quadratic TTA process.

The triplet-pair states formed can either dissociate back into independent triplets with rate $k_{D}$ or form a singlet state with rate $k_{T F}$, modulated by the singlet character $\left|C_{S}^{l}\right|^{2}$. The singlets decay radiatively with rate $k_{S}$. We also include an internal conversion channel, with overall rate $k_{I C}$, that results in the loss of one triplet; participation in this channel requires non-zero triplet character so the rate is modulated by $\left|C_{T}^{l}\right|^{2}$. Quintets simply break apart into independent triplets ${ }^{19,22}$, so we do not include them in the model. 
The rate equations describing these processes can be written as follows:

$$
\begin{aligned}
\frac{d\left[\mathrm{~T}_{1}\right]}{d t} & =G+2 k_{D} \sum_{l=1}^{9}\left[(\mathrm{TT})^{l}\right]+k_{I C} \sum_{l=1}^{9}\left|C_{T}^{l}\right|^{2}\left[(\mathrm{TT})^{l}\right] \\
& -2 k_{T T A}^{\prime}\left[\mathrm{T}_{1}\right] \\
\frac{d\left[(\mathrm{TT})^{l}\right]}{d t} & =\frac{1}{9} k_{T T A}^{\prime}\left[\mathrm{T}_{1}\right]-k_{D}\left[(\mathrm{TT})^{l}\right]-k_{I C}\left|C_{T}^{l}\right|^{2}\left[(\mathrm{TT})^{l}\right] \\
& -k_{T F}\left|C_{S}^{l}\right|^{2}\left[(\mathrm{TT})^{l}\right] \\
\frac{d\left[\mathrm{~S}_{1}\right]}{d t} & =k_{T F} \sum_{l=1}^{9}\left|C_{S}^{l}\right|^{2}\left[(\mathrm{TT})^{l}\right]-k_{S}\left[\mathrm{~S}_{1}\right] .
\end{aligned}
$$

Since $\Phi_{I S C}=\Phi_{T E T}=\Phi_{T T A}=\Phi_{P L}=1$ in this model, the spin statistical factor $\eta$ can be evaluated analytically by solving the equations under steady-state conditions. We obtain

$$
\begin{aligned}
\eta & =\frac{2 k_{S}\left[\mathrm{~S}_{1}\right]}{G} \\
& =\frac{\sum_{l=1}^{9} \frac{k_{T F}\left|C_{S}^{l}\right|^{2}}{k_{T F}\left|C_{S}^{l}\right|^{2}+k_{I C}\left|C_{T}^{l}\right|^{2}+k_{D}}}{\sum_{l=1}^{9} \frac{k_{T F}\left|C_{S}^{l}\right|^{2}+\frac{1}{2} k_{I C}\left|C_{T}^{l}\right|^{2}}{k_{T F}\left|C_{S}^{l}\right|^{2}+k_{I C}\left|C_{T}^{l}\right|^{2}+k_{D}}} .
\end{aligned}
$$

Equation (22) is identical to the expression previously arrived at by Schmidt and Castellano ${ }^{40}$, though it was not written out explicitly in their work. At the time however, the distinction between weak and strong exchange coupling within triplet-pair states was not so well understood, and the true implications were not fully grasped.

We can evaluate equation 22 for the limits of strongly and weakly exchange-coupled triplet-pairs discussed above. We find:

$$
\begin{aligned}
\eta_{\text {strong }} & =\left(1+\frac{3}{2} \frac{k_{I C} k_{T F}+k_{I C} k_{D}}{k_{I C} k_{T F}+k_{T F} k_{D}}\right)^{-1} \\
\eta_{\text {weak }} & =\left(1+\frac{1}{2} \frac{k_{I C} k_{T F}+3 k_{I C} k_{D}}{k_{I C} k_{T F}+k_{T F} k_{D}}\right)^{-1} .
\end{aligned}
$$

Assuming that the dissociation of triplet-pair states is considerably slower than fusion or internal conversion $\left(k_{D} \ll\right.$ $k_{T F}, k_{I C}$ ), we obtain, as expected ${ }^{20,40}, \eta=\frac{2}{5}$ in the limit of strong exchange coupling. Interestingly however, the spin statistical factor rises to $\eta=\frac{2}{3}$ for weakly exchange-coupled triplet pair states. In both cases, $\eta=1$ if $k_{I C}=0$.

We can understand these limits more intuitively by considering the probability tree associated with triplet-pair formation events (Fig. 2). Only triplet-pair states with singlet or triplet character are 'active' in TTA-UC and we let their probabilities of formation be $P_{S}$ and $P_{T}$ respectively. The spin statistical factor is then given by a geometric progression:

$$
\begin{aligned}
\eta & =P_{S}\left[1+\frac{1}{2} P_{T}+\left(\frac{1}{2} P_{T}\right)^{2}+\left(\frac{1}{2} P_{T}\right)^{3}+\ldots\right] \\
& =\frac{P_{S}}{1-\frac{1}{2} P_{T}} .
\end{aligned}
$$

In the case of strong exchange coupling, the relevant triplet-pair states comprise one pure singlet and three pure triplets giving $P_{S}=\frac{1}{4}$ and $P_{T}=\frac{3}{4}$, and hence $\eta=\frac{2}{5}$. For weakly exchange coupled triplet-pair states (on parallel molecules) we again have three pure triplets. The singlet character is spread across three singlet-quintet mixtures. The quintet component does not affect the fate of these mixed spin states, and so we have $P_{S}=P_{T}=\frac{1}{2}$ and therefore $\eta=\frac{2}{3}$.

Equations (22)-(24) allow us to identify the key factors expected to affect the spin statistics of TTA-UC. First, the inter-triplet exchange energy $J$ determines the character of the triplet-pair spin wavefunctions. If $J$ is negligible compared to other terms in the spin Hamiltonian (equation 5), the finer details of the intra-triplet spin dipolar interactions, including intermolecular orientation, also play a role. Second, the rates of internal conversion from ${ }^{3}$ (TT) to individual 


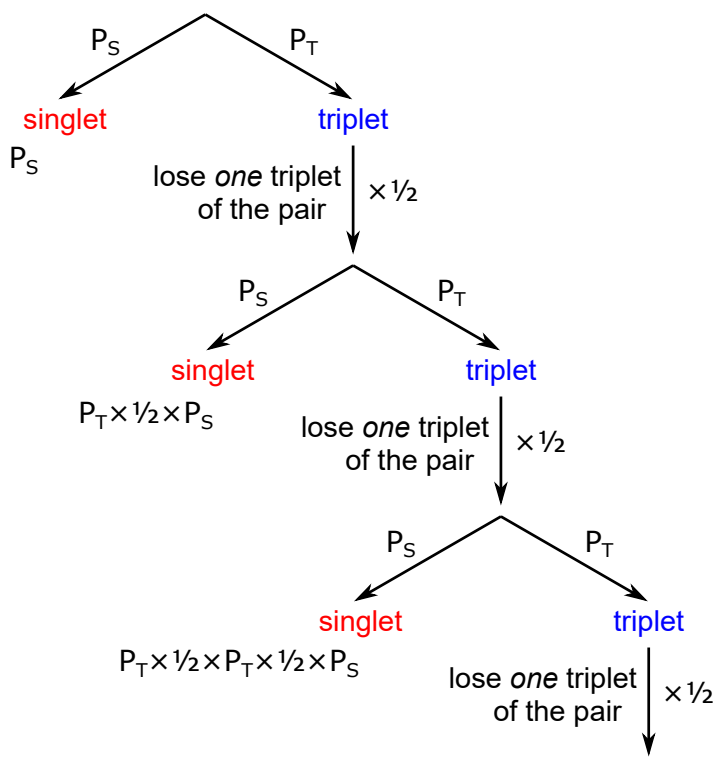

Figure 2. Probability tree for TTA spin statistics. The spin statistical factor can be evaluated using a probability diagram when the triplet character is contained exclusively in pure spin-1 states (there is no triplet-quintet or tripletsinglet mixing, as is the case for molecules oriented parallel). $P_{S}$ and $P_{T}$ are the respective probabilities of forming a triplet-pair state with singlet or triplet character.

triplet states $\mathrm{T}_{\mathrm{N}}$, and the subsequent fate of $\mathrm{T}_{\mathrm{N}}$, have a profound effect. If the internal conversion is slow in comparison to triplet-pair fusion and separation, or if high-level reverse intersystem crossing ${ }^{27,28,41,42}$ (HL-RISC) channels ${ }^{3}$ (TT) states to $S_{1}$ via $T_{2}$, the spin statistical factor can approach unity ${ }^{28}$. In the following, we investigate these factors in turn in the context of rubrene, the most common acceptor molecule for near-infrared-to-visible TTA-UC.

\section{Results}

Fig. 3a shows the molecular structure of rubrene. In crystalline rubrene, triplets are formed via singlet fission on the picosecond timescale ${ }^{43-45}$, allowing their fusion behaviour to be studied without the presence of sensitizer species ${ }^{46}$. We perform the majority of our experiments on rubrene nanoparticles (NPs) dispersed in a poly(vinyl alcohol) (PVA) matrix (Fig. 3b). Nanoparticles prepared in this way (see Experimental Section) have an average diameter of $220 \mathrm{~nm}$ and show no sharp peaks in their X-ray diffraction pattern ${ }^{47}$. These nanoparticle films are the basis of recently reported solid-state TTA-UC systems ${ }^{16,47}$.

In Fig. 3c we present the absorption and emission spectra of the rubrene NPs alongside the absorption spectrum of rubrene monomers in toluene. From these spectra, we confirm the $\mathrm{S}_{1}$ energy level at between $2.32 \mathrm{eV}$ and $2.23 \mathrm{eV}$ based on the absorption and emission maxima respectively. A small peak at $400 \mathrm{~nm}(3.1 \mathrm{eV})$ is clearly visible in the solution absorption spectrum which does not appear to follow the vibronic progression of the $S_{1}$ state. We suggest that this may be a signature of $S_{2}$ and that the strong absorption at around $300 \mathrm{~nm}(4.13 \mathrm{eV})$ corresponds to a higher-lying $\mathrm{S}_{0} \rightarrow \mathrm{S}_{\mathrm{N}}$ transition.

\section{Triplet-pair character}

Equations (22)-(24) demonstrate that the spin Hamiltonian of equation (5), in particular the inter-triplet exchange coupling $J$, has a profound effect on the spin statistical factor $\eta$. In order to probe the inter-triplet interactions in our rubrene NPs, we measured the effects of magnetic fields on the delayed fluorescence during bimolecular triplet-triplet annihilation.

Fig. 4a shows the time-resolved photoluminescence (PL) of a rubrene NP film at three different excitation intensities. Between $100 \mathrm{~ns}$ and $10 \mu \mathrm{s}$, we find that greater excitation density leads to a relative increase in measured PL. These 
a<smiles>c1ccc(-c2c3ccccc3c(-c3ccccc3)c3c(-c4ccccc4)c4ccccc4c(-c4ccccc4)c23)cc1</smiles>

b

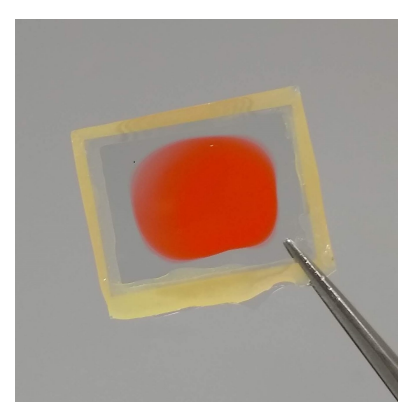

Energy $(\mathrm{eV})$

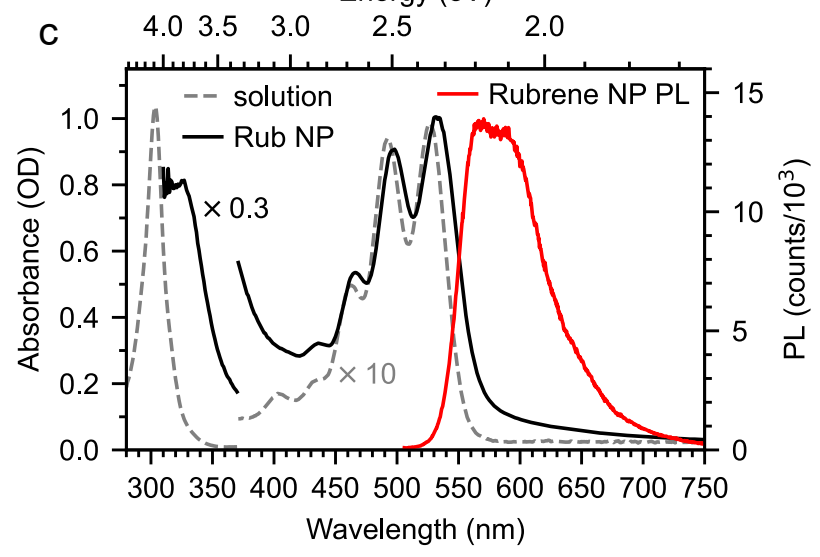

Figure 3. Rubrene nanoparticle films. (a) Molecular structure of rubrene. (b) Photograph showing a film of rubrene nanoparticles dispersed in an oxygen-blocking PVA matrix and cast onto a glass substrate. The sample is covered with a thin glass slip and sealed with epoxy resin. (c) Absorption and emission spectra of rubrene nanoparticle films alongside the absorption spectrum of rubrene dissolved in toluene $\left(1 \times 10^{-4} \mathrm{M}\right)$.

dynamics are characteristic of bimolecular triplet-triplet annihilation that, via triplet-pair intermediates, repopulates the $S_{1}$ state $^{26}$.

To investigate the character of the triplet-pair states that are the initial product of bimolecular TTA, in Fig. 4b we plot the change in PL intensity $0.5-1 \mu \mathrm{s}$ after excitation as a function of applied magnetic field, at the same three excitation intensities as Fig. 4a. We observe a small increase in the PL for fields $<50 \mathrm{mT}$ followed by a decrease at higher fields. The overall magnitude of the effect increases with excitation intensity, demonstrating that the tripletpairs responsible are products of bimolecular TTA.

Magnetic field effects (MFEs) such as those presented in Fig. 4b are well-known to be characteristic of triplet-triplet annihilation and were first explained by Johnson and Merrifield 50 years ago ${ }^{23,48}$. Their model for the spin physics of singlet fission and triplet-triplet annihilation is based on the spin Hamiltonian (equation 5) but with no exchange term, i.e. $J=0$. Thus Johnson and Merrifield's rather vaguely defined 'TT' states are implicitly weakly exchange-coupled, though such terminology was not used at the time. MFEs measured under fields of a few tens of $\mathrm{mT}$ are therefore signatures of weakly exchange-coupled triplet-pair states ${ }^{35}$. This can be readily understood by examining the spin Hamiltonian of equation (5).

The zero-field splitting parameter $D$ is typically around $10 \mu \mathrm{eV}$. For example, it is $6.45 \mu \mathrm{eV}$ in tetracene ${ }^{49}$ and is thought to be similar for rubrene ${ }^{29}$. The Zeeman term thus has a similar magnitude to the zero-field term when $g \mu_{B} B \sim D$, i.e. $B \sim 50 \mathrm{mT}$. In the absence of other terms in the spin Hamiltonian of similar or greater magnitude, the competition between the Zeeman and zero-field terms at such fields leads to variations in the eigenstates $\left|\psi_{l}\right\rangle$ with magnetic field and hence to variations in the singlet character $\left|C_{S}^{l}\right|^{2}$ (equation 15) of the triplet-pair states ${ }^{23,38}$. For example we have seen that when $B=0$, three of the eigenstates $(|x x\rangle,|y y\rangle$ and $|z z\rangle)$ have singlet character. If $g \mu_{B} B \gg D$, this falls to two ${ }^{23}$, giving rise to the characteristic reduction in measured PL during triplet-triplet annihilation. If however, as is implicitly assumed in discussions of spin statistics for TTA-UC, the triplet-pairs formed are strongly exchange-coupled $(J \gg D)$, we would not see any significant MFE until $g \mu_{B} B \sim J$, since the zero-field term now acts only as a tiny perturbation. This requires much higher field strengths and gives rise to very different 

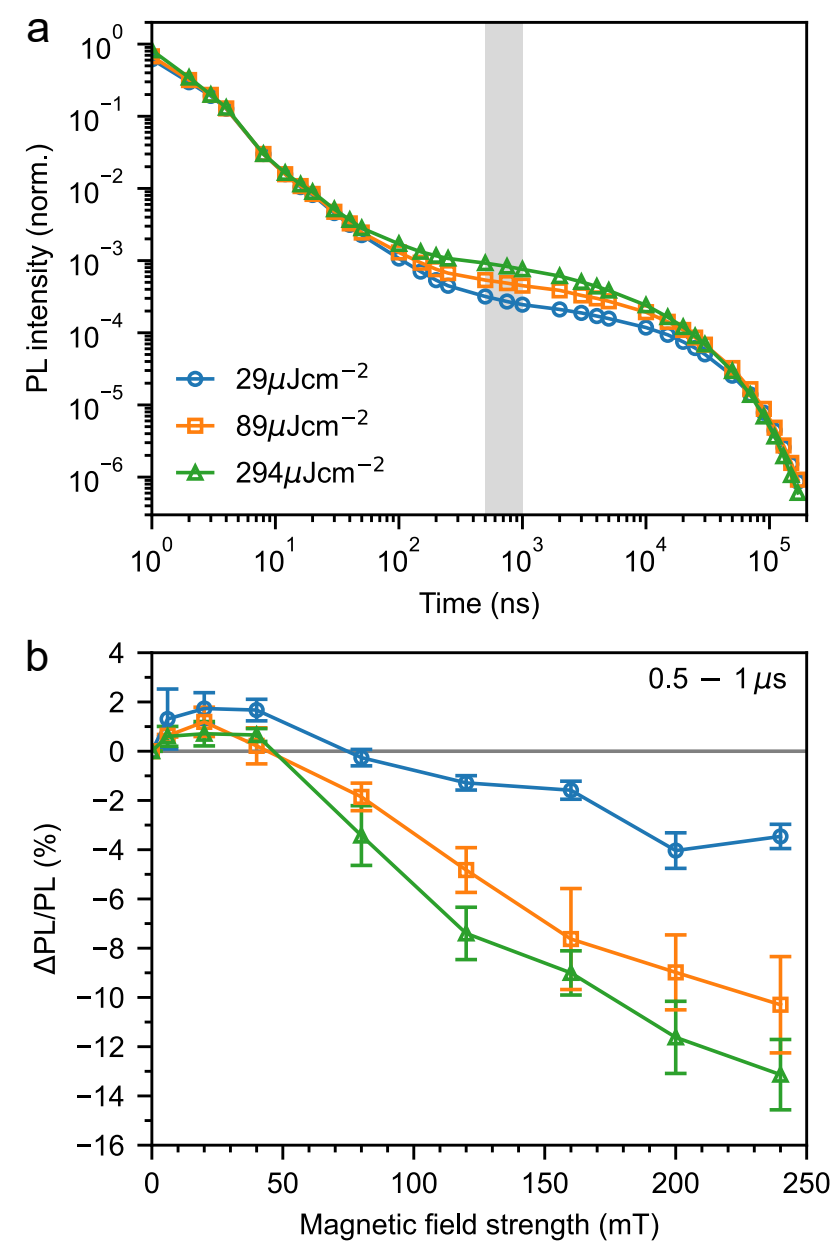

Figure 4. Triplet-triplet annihilation and magnetic field effects. (a) Time-resolved photoluminescence (PL) of a rubrene nanoparticle film at three different excitation intensities. The decays have been normalised at $8 \mathrm{~ns}$. (b) Magnetic field effects (MFEs) on fluorescence gated from $0.5-1 \mu \mathrm{s}$ at the same three excitation intensities. Error bars reflect the variation between sweeping up and down in magnetic field and arise from slight photobleaching and small fluctuations in laser power.

types of MFE ${ }^{35,37}$.

MFEs corresponding to weakly exchange-coupled triplet-pairs, similar to ours in Fig. 4b, have been measured during TTA-UC both in the solid state ${ }^{39}$ and in solution ${ }^{50,51}$. We have explained why such MFEs are evidence that the triplet-pairs first formed through bimolecular TTA are weakly exchange-coupled. Below, we explore the implications of this for the spin statistics of TTA-UC. First however, we investigate the other key factors that may impact the spin statistical factor: internal conversion, energy levels and reverse-intersystem crossing.

\section{Energy levels and internal conversion}

In order to estimate the rates of internal conversion from ${ }^{3}$ (TT) to $\mathrm{T}_{\mathrm{N}}$, we must first determine the triplet energy levels. The energy of $\mathrm{T}_{1}$ is well known to be $1.14 \mathrm{eV}$ for rubrene ${ }^{13,52,53}$. We can therefore take the energy of ${ }^{3}$ (TT) to be $2.28 \mathrm{eV}$ in the absence of large inter-triplet binding. Reported values for the rubrene $\mathrm{T}_{2}$ energy vary significantly ${ }^{54-57}$. For a precise determination of the higher lying triplet energies, we turn to transient absorption (TA) spectroscopy.

Fig. 5 shows transient absorption spectra of a rubrene NP film pumped at $532 \mathrm{~nm}$. We find the characteristic signatures of singlet fission in rubrene: the singlet photo-induced absorption (PIA) at $440 \mathrm{~nm}$ decays rapidly, accompanied by a rise in the triplet PIA at $510 \mathrm{~nm}^{43}$. Broad PIA features at around $680 \mathrm{~nm}$ and $1170 \mathrm{~nm}$ decay with similar dynamics to the $440 \mathrm{~nm}$ band (Fig. S1) and we therefore assign them to $S_{1} \rightarrow S_{N}$ transitions, as reported previously ${ }^{45,53}$. Finally, we observe two PIA peaks in the near-infrared at $960 \mathrm{~nm}$ and $850 \mathrm{~nm}$ (Fig. 5c) whose dynamics match those of the well-known triplet PIA at $510 \mathrm{~nm}$ (Fig. S1). Similar peaks have previously been assigned to triplet states in 


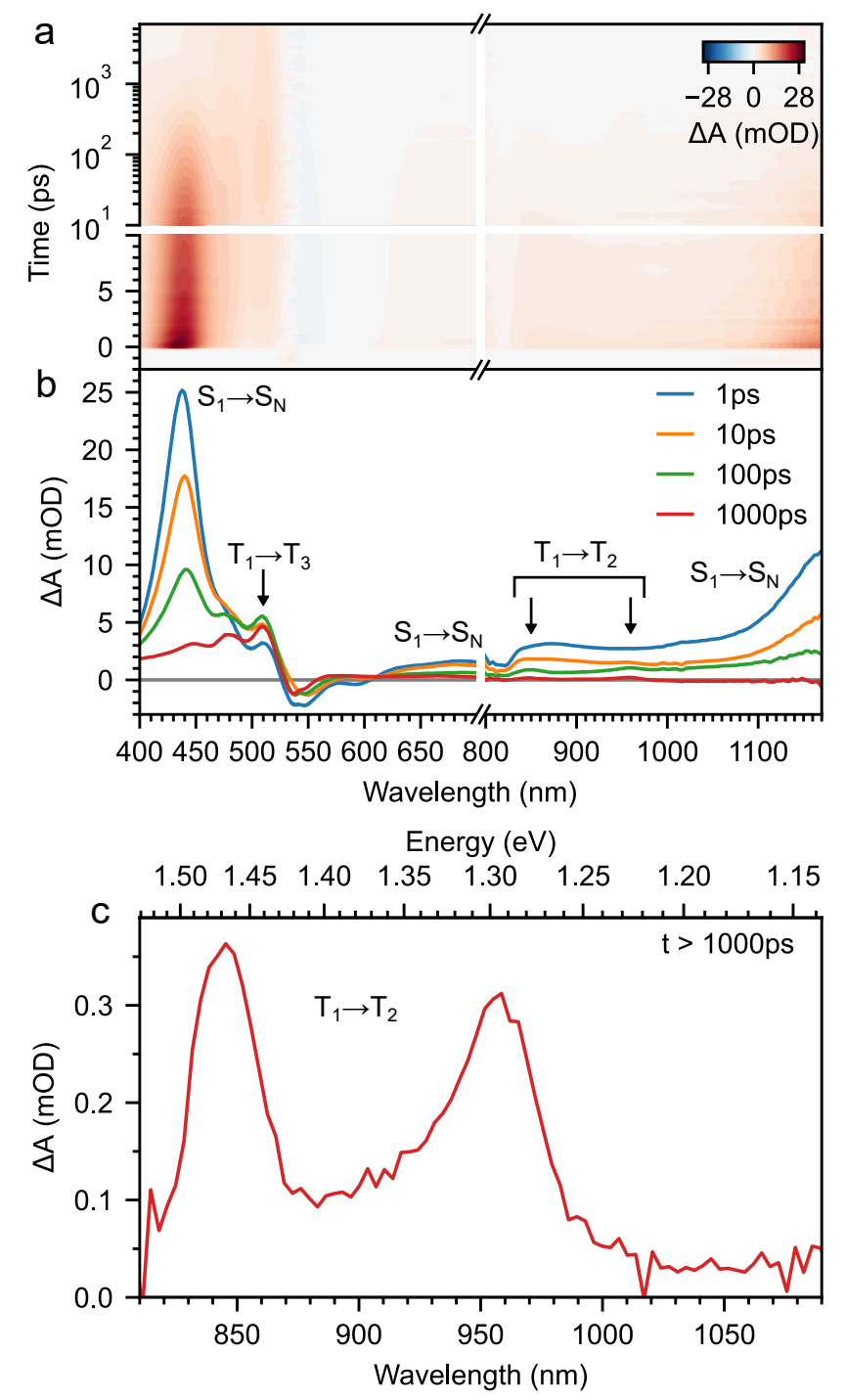

Figure 5. Transient absorption spectroscopy of rubrene nanoparticle films. (a) False-colour map showing transient absorption measurements of rubrene NP films pumped at $532 \mathrm{~nm}$ with an excitation intensity of $40 \mu \mathrm{J} \mathrm{cm}^{-3}$. (b) Transient absorption spectra spanning the visible and near-infrared reveal singlet fission dynamics. Singlet photoinduced absorption (PIA) features at $440 \mathrm{~nm}, 680 \mathrm{~nm}$ and $1170 \mathrm{~nm}$ decay, accompanied by a rise in triplet PIA bands at $510 \mathrm{~nm}, 850 \mathrm{~nm}$ and $960 \mathrm{~nm}$. The latter two peaks, highlighted in (c), correspond to the $0-0$ and $0-1$ bands of the $\mathrm{T}_{1} \rightarrow \mathrm{T}_{2}$ transition. Given the $\mathrm{T}_{1}$ energy of $1.14 \mathrm{eV}$, we calculate the $\mathrm{T}_{2}$ and $\mathrm{T}_{3}$ energy levels to be $2.43 \mathrm{eV}$ and $3.57 \mathrm{eV}$ respectively.

rubrene $^{53}$. Broad PIA features in the same spectral region have been explicitly assigned to $T_{1} \rightarrow T_{2}$ transitions in rubrene $^{45}$, in agreement with calculations ${ }^{58}$. The two sharp peaks that we measure here are separated in energy by $0.17 \mathrm{eV}$, suggesting that they belong to a vibronic progression. We therefore assign them to the $0-0$ and $0-1$ vibronic peaks of the $T_{1} \rightarrow T_{2}$ transition, putting the $T_{2}$ energy at $2.43 \mathrm{eV}$. The next triplet PIA is that at $510 \mathrm{~nm}$, suggesting that $\mathrm{T}_{3}$ lies at $3.57 \mathrm{eV}$.

We use the photo-induced absorptions from Fig. 5 to construct the energy level diagram of rubrene shown in Fig. 6a. Of particular importance for the spin statistics of upconversion are the energy differences between $2 \mathrm{~T}_{1} \approx$ ${ }^{3}(\mathrm{TT}), \mathrm{T}_{1}$ and $\mathrm{T}_{2} \cdot{ }^{3}$ (TT) $\rightarrow \mathrm{T}_{1}$ is exothermic by $1.14 \mathrm{eV}$, whilst ${ }^{3}(\mathrm{TT}) \rightarrow \mathrm{T}_{2}$ is endothermic by $150 \mathrm{meV}=6 k_{B} T$. To date, only the relative energy levels have been considered in determining whether the ${ }^{3}$ (TT) $\rightarrow \mathrm{T}_{\mathrm{N}}$ loss channel is operational in TTA-UC ${ }^{14}$. Here, we aim to go a step further by estimating the rates of the internal conversions.

In the absence of strong vibronic or non-adiabatic coupling, the rate of internal conversion in organic molecules obeys the energy gap law ${ }^{61}$, which we write as

$$
k_{I C}=A \exp \left(-\frac{\gamma \Delta E}{\hbar \omega_{0}}\right)
$$



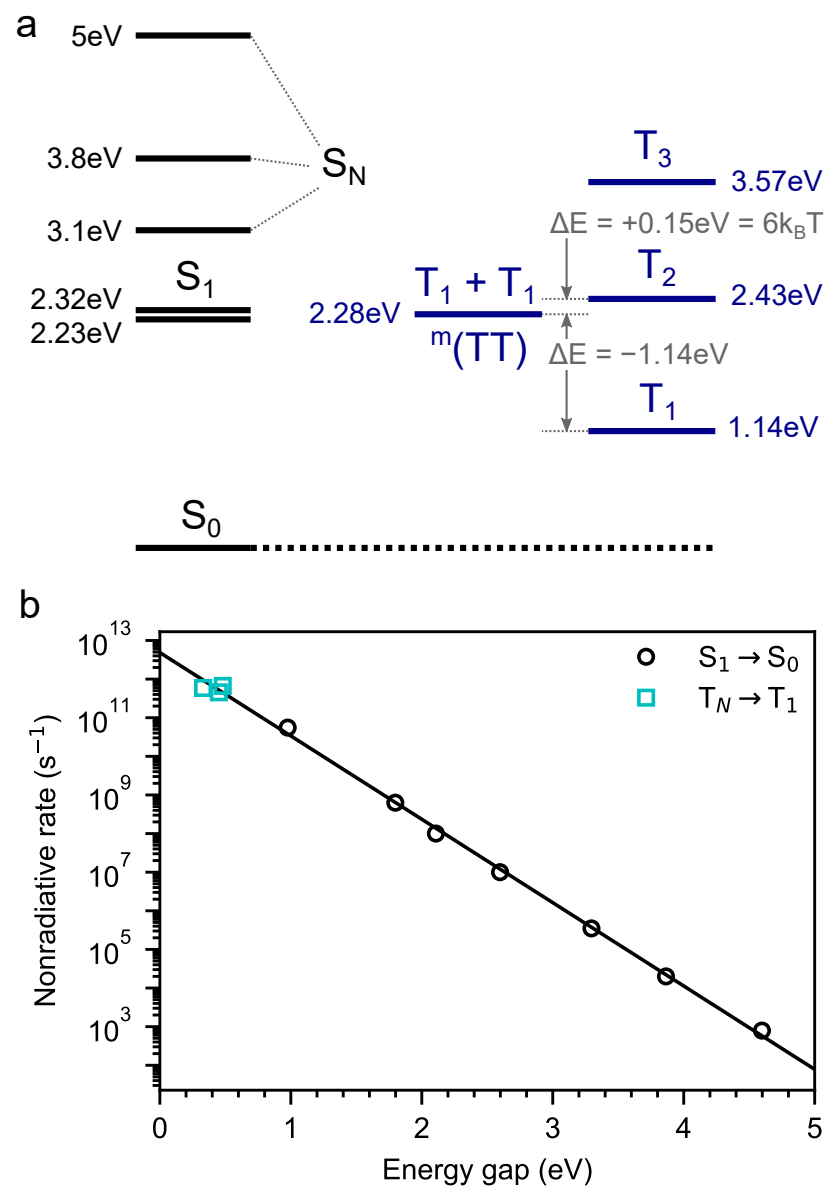

Figure 6. Energy levels of rubrene and internal conversion in acenes. (a) Energy level diagram for rubrene based on the transient absorption spectra in Fig. $5 .{ }^{3}$ (TT) $\rightarrow \mathrm{T}_{1}$ is exothermic by 1.14 and ${ }^{3}$ (TT) $\rightarrow \mathrm{T}_{2}$ is endothermic by $150 \mathrm{meV}=6 k_{B} T$. (b) $\mathrm{S}_{1} \rightarrow \mathrm{S}_{0}$ nonradiative rates plotted against optical gap for acenes based on data in Refs. ${ }^{59}$ and ${ }^{60}$. We find excellent agreement with the energy gap law. Measurements of triplet-triplet internal conversion rates in erythrosin B, rose bengal and tetraphenylporphyrin ${ }^{41}$ follow the same gap law.

where $\Delta E$ is the energy gap between the electronic states, $\hbar \omega_{0}$ is the highest available vibrational frequency that couples to the electronic states (taken to be the symmetric vinyl stretching mode at $0.17 \mathrm{eV}$ ) ${ }^{62}$ and $\gamma$ and the prefactor $A$ are material system dependent.

We begin by assuming that internal conversion in the triplet and singlet manifolds obeys the same energy gap law. For singlet internal conversions, we use the rate of the nonradiative $S_{1} \rightarrow S_{0}$ transition. This has been determined experimentally for the acene family from benzene through to hexacene ${ }^{59}$ and also for carbon nanotubes ${ }^{60}$. Following Ref. 25, we plot these internal conversion rates against their optical gaps in Fig. 6b and find excellent correspondence with the energy gap law. This allows us to extract values of $A=(4.9 \pm 1.3) \times 10^{12} \mathrm{~s}^{-1}$ and $\gamma=0.845 \pm 0.015$ for molecules comprising fused aromatic rings. Experimental determinations of triplet-triplet internal conversions are much less common, though measurements do exist for erythrosin B, rose bengal and tetraphenylporphyrin ${ }^{41}$. Plotting these values on Fig. 6b, we find good agreement with the energy gap law for singlet manifold internal conversions, providing some justification of our earlier assumption.

We use the values of $A$ and $\gamma$ extracted from Fig. $6 \mathrm{~b}$ in equation (27) to estimate the triplet internal conversion rates in rubrene. For the exothermic ${ }^{3}(\mathrm{TT}) \rightarrow \mathrm{T}_{1}$ process, we find a rate of $(1.7 \pm 0.5) \times 10^{10} \mathrm{~s}^{-1}$ or $(60 \pm 20) \mathrm{ps}$. The endothermic route via $\mathrm{T}_{2}$ requires thermal activation, but can then proceed with an energy gap of zero. Thus the rate can be approximated by

$$
k_{3}(\mathrm{TT}) \rightarrow \mathrm{T}_{2}=A \exp \left(-\frac{\Delta E_{\mathrm{T}_{2}-3}(\mathrm{TT})}{k_{B} T}\right),
$$

which evaluates to $(1.2 \pm 0.3) \times 10^{10} \mathrm{~s}^{-1}$ or $(80 \pm 20) \mathrm{ps}$ at room temperature. The internal conversion rates are therefore expected to be similar for transitions to $\mathrm{T}_{1}$ and $\mathrm{T}_{2}$ despite the endothermic nature of the latter. This is highly 
significant: it has been recently reported that high-level reverse intersystem crossing (HL-RISC) from $T_{2}$ to $S_{1}$ can occur in rubrene ${ }^{27}$, potentially providing a pathway from ${ }^{3}$ (TT) to $S_{1}$ that could alleviate at least some of the losses usually implied by the formation of ${ }^{3}(\mathrm{TT})^{28}$. We therefore use pump-push-probe spectroscopy to investigate the fate of the $\mathrm{T}_{2}$ state in rubrene.

\section{High-level reverse intersystem crossing}
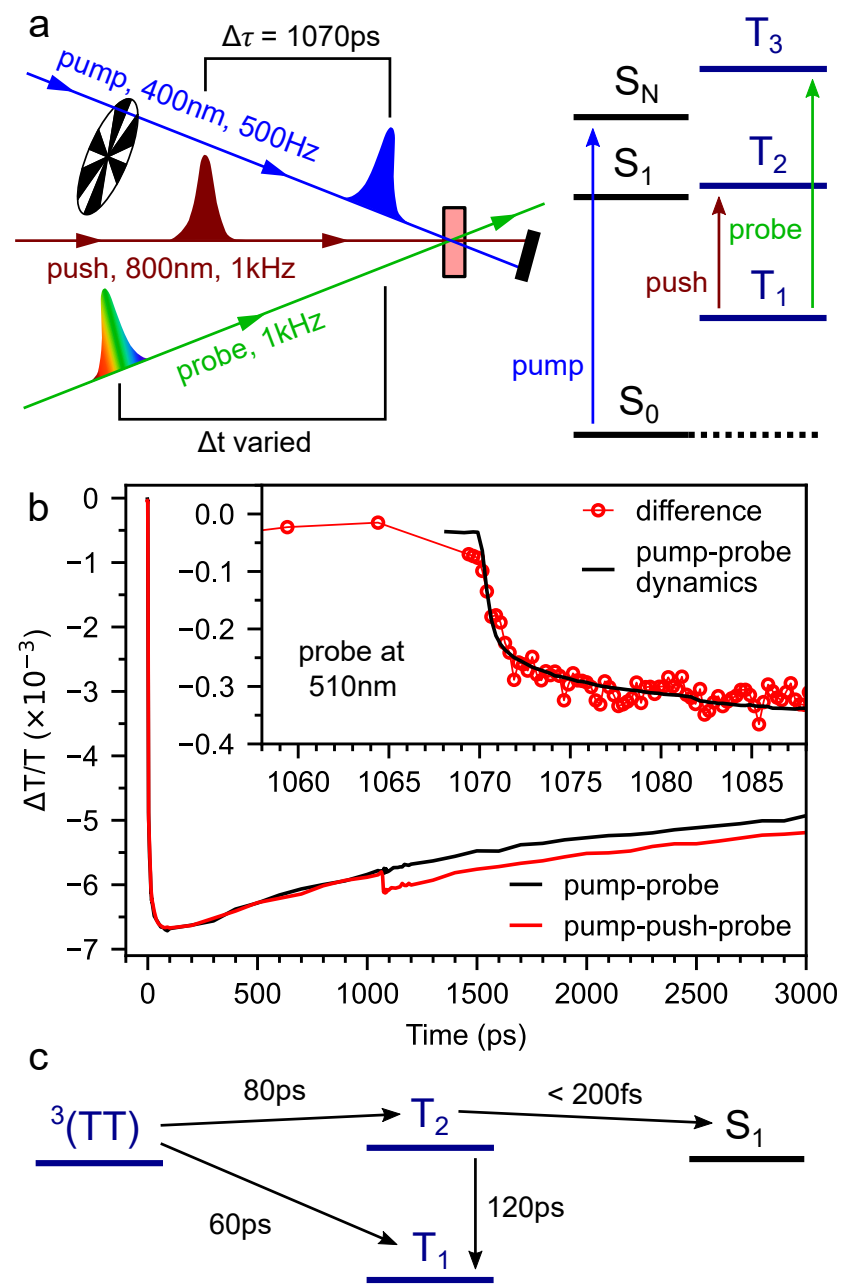

Figure 7. Pump-push-probe spectroscopy of rubrene. (a) Illustration of the pump-push-probe experiment and the electronic transitions targeted by each pulse. The pump initiates singlet fission. After $1 \mathrm{~ns}$, the excited state population will be principally triplets, which are excited from $T_{1}$ to $T_{2}$ by the sub-bandgap push pulse. The probe is used to investigate the effect of the push pulses with and without the initial pump. (b) Pump-probe and pump-push-probe data recorded at a probe wavelength of $510 \mathrm{~nm}$ (the $T_{1} \rightarrow T_{3}$ transition) for a polycrystalline rubrene thin film. The push pulse causes an enhancement of the $\mathrm{T}_{1} \rightarrow \mathrm{T}_{3}$ photo-induced absorption, with dynamics that match the initial singlet fission. (c) Interpretation of the pump-push-probe data in terms of high-level reverse intersystem crossing from $\mathrm{T}_{2}$ to $\mathrm{S}_{1}$.

Fig. 7a illustrates the pump-push-probe experiment and the transitions in rubrene targeted by each pulse. The $400 \mathrm{~nm}$ pump pulses photo-excite the singlet manifold, thereby initiating singlet fission. The push pulses are delayed by a constant $1 \mathrm{~ns}$ with respect to the pump, by which time triplets are expected to be the dominant excited states. The sub-bandgap $800 \mathrm{~nm}$ push pulses are approximately resonant with the $\mathrm{T}_{1} \rightarrow \mathrm{T}_{2}$ transition $^{56}$ and we monitor the probe transmission at $510 \mathrm{~nm}$, which corresponds to $T_{1} \rightarrow T_{3}$. Other probe wavelengths show no discernable pushinduced effects due to reduced signal to noise (the triplet PIA is sharply peaked at around $510 \mathrm{~nm}$ ). These are shown in Supplementary Fig. S3. We halve the frequency of the pump pulses only, and record the differential transmission as a function of pump-probe delay. 
We performed the pump-push-probe experiment on a polycrystalline thin film (characterisation in Supplementary Fig. S2) rather than the rubrene NPs, since we found it to possess a stronger triplet excited state absorption at $510 \mathrm{~nm}$, giving sufficient signal to noise to measure the push-induced effects. Fig. 7b shows the results with (red) and without (black) the presence of the push pulses. We find that the push from $T_{1}$ to $T_{2}$ causes an increase, rather than a bleach, of the $\mathrm{T}_{1}$ population. Furthermore, the dynamics of the push induced enhancement match the regular pump-probe dynamics of singlet fission, as shown in the inset of Fig. 7b.

We consider several possibilities for the underlying photophysics, which we discuss in detail in Supplementary Information Section 2.1. First, if the $\mathrm{T}_{2}$ states populated by the push pulses simply undergo internal conversion to $\mathrm{T}_{1}$, we would expect to see a bleach, and subsequent recovery of the $\mathrm{T}_{1}$ population. Alternatively, the push could act as a second pump, perhaps through two-photon absorption ${ }^{63,64}$. In this case, the ground state population available to be 're-pumped' by the push is depleted by the first pump pulse, and we would again expect to see a reduction in signal when the push is present. Instead, we observe an enhancement.

We suggest that these results are consistent with recent reports of high-level reverse intersystem crossing from $\mathrm{T}_{2}$ to $S_{1}$ in rubrene ${ }^{27}$. In this case, $T_{2}$ states populated by the push are converted, via $S_{1}$ and singlet fission, into pairs of triplets. This can only occur in the presence of the initial pump; it therefore manifests itself as an enhancement in the triplet signal rather than a bleach, since each $\mathrm{T}_{2}$ state results in a pair of triplets. We note that the reverse intersystem crossing must occur within the instrument response of our setup ( $\sim 200 \mathrm{fs})$ for this explanation, which we summarise in Fig. 7c, to be consistent with our results.

There is precedent for expecting HL-RISC to occur in rubrene. It is well known that thermally activated intersystem crossing from $S_{1}$ to $T_{2}$ occurs in rubrene ${ }^{54,55}$, though estimates of the Arrhenius parameters differ by several orders of magnitude between measurements in solution ${ }^{54}$ and solid glasses ${ }^{55}$. It must also be possible therefore for the exothermic HL-RISC process to occur. Furthermore, HL-RISC was proposed by several authors to explain high TTA-UC efficiencies in OLED devices based on rubrene ${ }^{65}$ and substituted anthracenes ${ }^{28,42,66}$ (though it is interesting to note that it does not occur in diphenylanthracene (DPA) ${ }^{28}$, perhaps due to symmetry restrictions ${ }^{67}$ ). Recently, a detailed study of magnetic field effects in rubrene-based OLEDs confirmed that HL-RISC was occurring ${ }^{27}$. The sub-picosecond timescale is also plausible: HL-RISC rates for erythrosin B, rose bengal and tetraphenylporphyrin have been measured to be $1 \mathrm{ps}$ or less ${ }^{41}$. The $\mathrm{S}_{1}-\mathrm{T}_{2}$ energy gaps in these three dyes are several hundred meV greater than in rubrene, so we might expect the HL-RISC rate in the latter to be even faster. Finally, we note that vibronic coupling effects have been calculated to increase RISC rates by several orders of magnitude in thermally activated delayed fluorescence (TADF) molecules ${ }^{68}$ and suggest that similar effects could help to enable sub-picosecond HL-RISC in rubrene.

\section{Discussion}

Given that the initial products of TTA are weakly exchange-coupled triplet pairs, and given the important distinction between internal conversions from ${ }^{3}$ (TT) to $\mathrm{T}_{1}$ and $\mathrm{T}_{2}$, we can extend our simple scheme (model 1) from Fig. 1 into that shown in Fig. 8 (model 2). Now we explicitly differentiate between triplet-pair states (T...T) ${ }^{l}$ formed through TTA, which are governed by the spin Hamiltonian in equation (5), and the pure spin states ${ }^{1}$ (TT), ${ }^{3}$ (TT) and ${ }^{5}$ (TT), which couple to the (T...T $)^{l}$ states through their singlet, triplet and quintet character respectively. In the limit of $J \gg D$, these two sets of states will coincide. We also include a singlet fission channel, and add a distinct $\mathrm{T}_{2}$ state that is permitted to undergo HL-RISC to form $\mathrm{S}_{1}$.

The rate equations governing this extended model are given in the Supplementary Information. The upconversion quantum yield can be calculated from

$$
\Phi_{U C}=\frac{k_{S}\left[S_{1}\right]}{G_{T}},\left(G_{S}=0\right),
$$

whilst the photoluminescence quantum yield is given by

$$
\Phi_{P L}=\frac{k_{S}\left[\mathrm{~S}_{1}\right]}{G_{S}},\left(G_{T}=0\right),
$$

where $G_{T}$ and $G_{S}$ are the generation rates for triplet and singlet states respectively. If the rate of singlet fission $k_{S F}$ is non-zero, $\Phi_{P L}$ may not be unity and instead will depend on the spin statistical factor $\eta$, which in general can be 


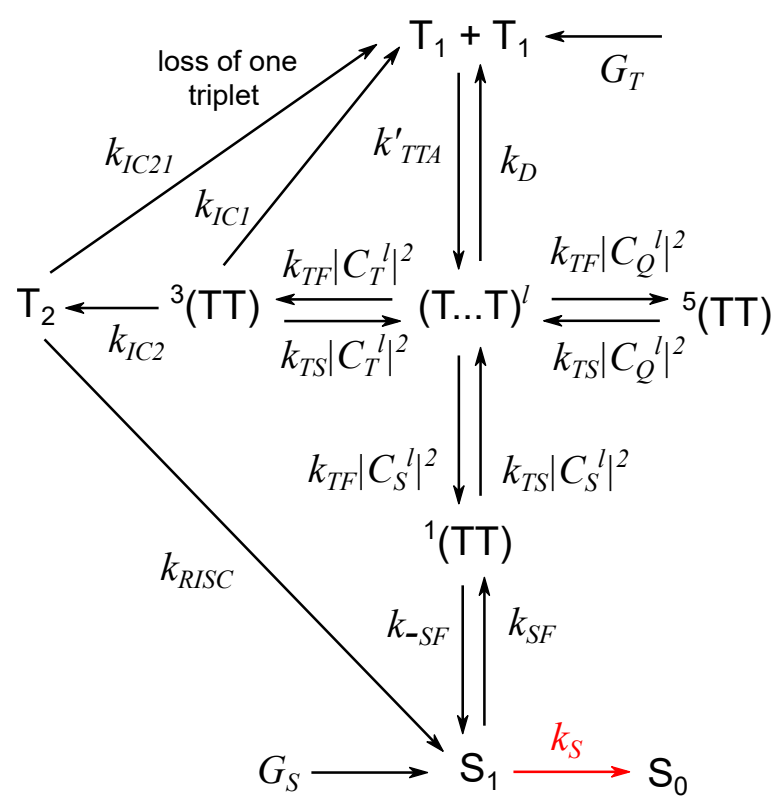

Figure 8. An extended model of TTA-UC. Schematic diagram showing a refinement of the model in Fig. 1. We differentiate between triplet-pairs formed directly through TTA, (T...T), and strongly-exchange coupled pure spin triplet-pairs, ${ }^{1 / 3 / 5}$ (TT). In the limit of strong exchange coupling, these sets of states are identical. We also include a singlet fission channel, and provide two distinct internal conversion channels from ${ }^{3}$ (TT). $\mathrm{T}_{2}$ can undergo HL-RISC to form $S_{1}$. This is referred to as model 2 .

calculated as

$$
\eta=\frac{2 \Phi_{U C}}{\Phi_{P L}} .
$$

Order-of-magnitude values for the main rate constants are given in Supplementary Table S1. As shown Supplementary Fig. S4, the values of $k_{T T A}^{\prime}$ and $k_{S F}$ have no effect on the model predictions and neither does $G_{i=S \text { or } T}$ since the equations are linear. The other rate constants can be varied significantly from the values in Supplementary Table S1 with little impact. Large variations in $k_{T F}, k_{D}$ and $k_{I C}$ do have an effect on $\eta$ but this is only to be expected ${ }^{40}$ from equation 22. We thus consider the conclusions drawn from the model to be robust and highly general. In our simulations, we use the zero-field splitting parameters of tetracene ${ }^{49}, D=6.45 \times 10^{-6} \mathrm{eV}$ and $E=-6.45 \times 10^{-7} \mathrm{eV}$ and take $X=D / 1000$.

Fig. 9 shows the key predictions from the model. In order to investigate the effects of inter-triplet exchange coupling, we begin by switching off the HL-RISC channel and taking the simplest case of parallel molecules, common to most acene crystals including rubrene. Next, we explore the effects of non-parallel molecular orientation and finally we introduce the HL-RISC channel.

Fig. 9a shows the simulated MFE for triplet-triplet annihilation in the limits of strong (red) and weak (blue) exchange coupling. To demonstrate the generality of our model, we also show the (identical) predictions from model 1 , incorporating a singlet fission channel (circles). As expected, we find that only in the limit of weak exchange coupling between triplets following TTA do we reproduce the experimentally measured MFE (Fig. 4b). The lower panel of Fig. 9a illustrates the origin of the $J=0$ MFE by plotting the number of (T...T) $)^{l}$ states with $\left|C_{S}^{l}\right|^{2}>5 \%$ (i.e. more than $5 \%$ singlet character) as a function of magnetic field, along with equivalent numbers for triplet and quintet character. The threshold of $5 \%$ was chosen because it nicely illustrates the key behaviours. At higher fields, two rather than three of the (T...T) $)^{l}$ triplet-pair states have appreciable singlet character, leading to reduced PL. We note that the HL-RISC channel would introduce further magnetic field effects: the $S_{1}$ states formed can undergo singlet fission, which gives an inverted MFE shape compared to TTA, and the RISC process itself carries a (negative) magnetic field effect $^{27}$ which is beyond the scope of our model.

In Fig. 9b we plot the spin statistical factor for TTA-UC as a function of inter-triplet exchange energy. In the conventionally assumed but as we have explained, incorrect, case of strong exchange coupling we find the expected limit of $\eta=\frac{2}{5}$. As shown in the lower panel, this is the case for eigenstates that are pure spin states: 5 quintets, 3 

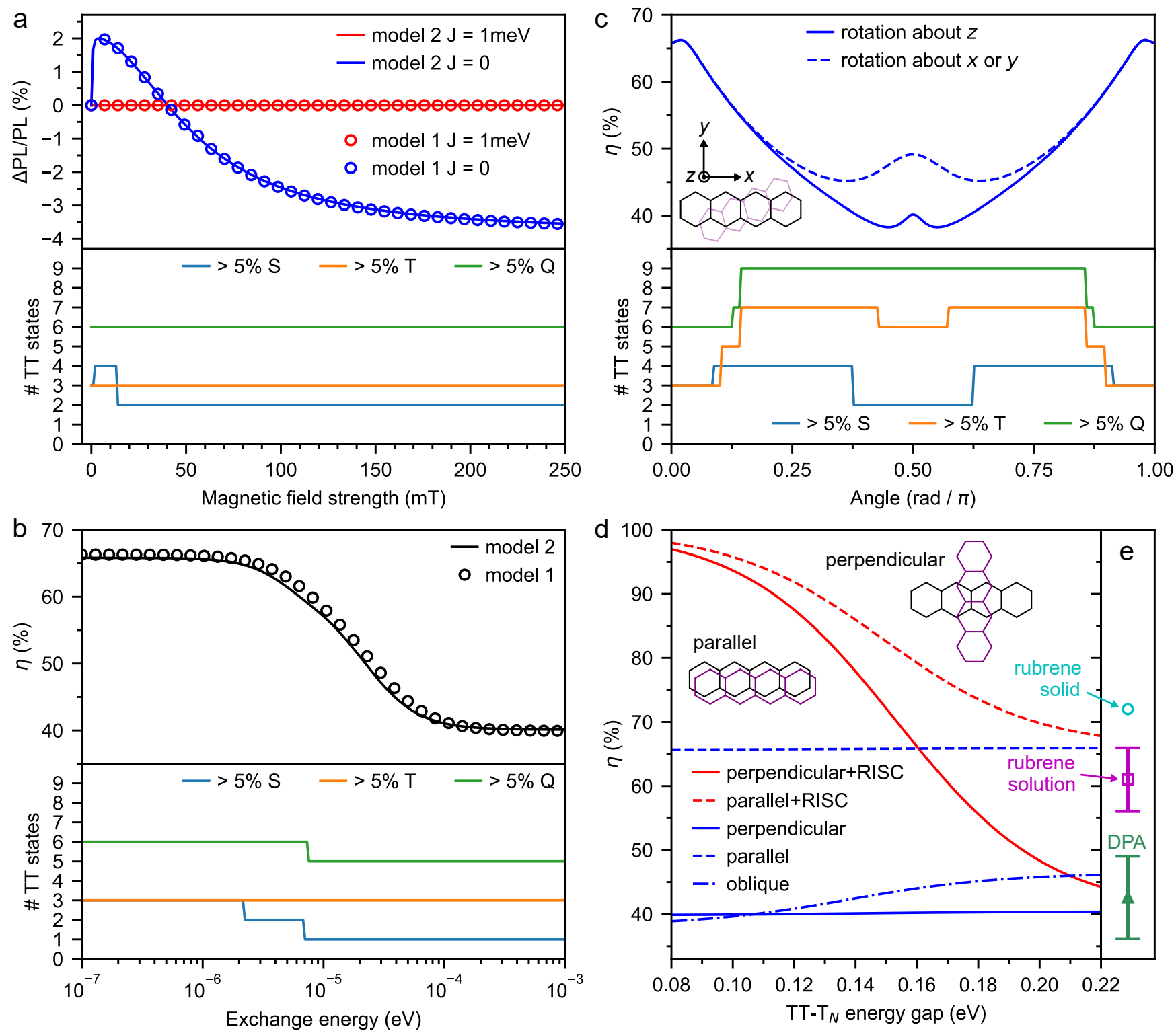

Figure 9. Simulations of factors controlling the spin statistics of TTA-UC. (a) Simulated MFE for parallel molecules comparing model 1 (Fig. 1) and model 2 (Fig. 8) for strongly $(J=1 \mathrm{meV})$ and weakly $(J=0)$ exchange-coupled triplet-pairs. The lower panel shows changes in spin character of the $J=0$ triplet-pairs with applied magnetic field. Note that $>5 \% \mathrm{~S}$ means triplet-pair states with $\left|C_{S}^{l}\right|^{2}>5 \%$, i.e. more than $5 \%$ singlet character, and similarly for triplet (T) and quintet (Q) character. The simulation shows that the experimental MFE (Fig. 4b) can only be reproduced if the initial products of TTA are weakly exchange-coupled triplet-pairs. (b) Simulated spin statistical factor $\eta$ for parallel molecules as a function of inter-triplet exchange energy $J$. The lower panel again shows the changes in triplet-pair spin character. The simulation shows that, for parallel molecules and in the absence of HL-RISC, $\eta=\frac{2}{3}$ rather than the conventionally assumed $\frac{2}{5}$. (c) Model 2 simulation showing the variation of $\eta$ with inter-molecular orientation, for $J=0$ and $k_{R I S C}=0$. The lower panel shows triplet-pair spin character. Changes to singlet-triplet-quintet spin mixing causes $\eta$ to vary between $\frac{2}{3}$ (parallel orientation) and $\frac{2}{5}$ (perpendicular orientation). (d) Model 2 simulation of $\eta$ as a function of ${ }^{3}$ (TT)- $\mathrm{T}_{\mathrm{N}}$ energy gap (for rubrene, $N=2$ ), for several different cases, all with $J=0$. The presence of HL-RISC causes a sensitive dependence on $\mathrm{T}_{2}$ energy, relative to ${ }^{3}$ (TT), with smaller gaps leading to higher values of $\eta$. In the absence of HL-RISC, this dependence is much weaker and shows the opposite trend. The oblique case, corresponding to an inter-molecular geometry found in the DPA crystal ${ }^{69}$, shows a more pronounced dependence, due to increased triplet-singlet and triplet-quintet mixing and hence greater variation in the internal conversion rates. These simulations show that differences in triplet-pair orientation, coupled with the presence or absence of HL-RISC, can explain the different values of $\eta$ measured experimentally for rubrene and DPA, shown in (e). (e) Reported experimental ranges of $\eta$ for DPA, rubrene in solution and rubrene in the solid state, obtained from Refs. ${ }^{17,18,21,28,53,54,65,70-76}$. These experimental values, together with reported values of the rubrene $\mathrm{T}_{2}$ energy level, are given in Supplementary Tables S2 and S3 respectively. 
triplets and 1 singlet. The spin statistical factor rises to $\frac{2}{3}$ as the exchange coupling is reduced, reflecting the increase (from 1 to 3 ) in the number of triplet-pair states possessing significant singlet character.

As discussed above, the spin character of weakly exchange-coupled triplet-pair states is dependent on the relative orientation of the two molecules involved ${ }^{29,32,35}$. This has a knock-on effect on the spin statistical factor, as shown in Fig. 9c. Rotation of one molecule of the pair with respect to the other causes increased singlet-triplet-quintet mixing. In particular, the greater number of states possessing significant triplet character results in a higher probability for ${ }^{3}(\mathrm{TT}) \rightarrow \mathrm{T}_{\mathrm{N}}$ internal conversions, thereby reducing the spin statistical factor (in the absence of efficient HL-RISC). The dependence of $\eta$ on relative molecular orientation may help to explain differences in TTA efficiency between monomeric annihilators and rigid dimers ${ }^{77}$. Furthermore, it introduces an important consideration for the design of solid state upconversion systems. We find that the parallel orientations associated with close $\pi-\pi$ stacking (and hence rapid triplet diffusion ${ }^{78}$ ) in acene crystals also result in the best spin statistical factors.

Finally, in Fig. 9d, we explore the impact of HL-RISC on the spin statistical factor by plotting $\eta$ against $\mathrm{T}_{2}$ energy (relative to the ${ }^{3}$ (TT) level) for several different cases. In solution, the common annihilator molecules rubrene and DPA are thought to form triplet-pair complexes in which the chromophores are oriented perpendicular to each other ${ }^{40}$. In this case, the spin-statistical factor is $40 \%$ in the absence of a HL-RISC channel, but we emphasise that this is a result of weakly interacting triplet-pair states with mixed singlet, triplet and quintet character and not because TTA forms pure singlet, triplet and quintet complexes in a 1:3:5 ratio. We suggest that this is the reason that DPA in solution is reported to give $\eta \sim 40 \% 8,9,28,40,70-74$. The range of experimentally measured values of $\eta$ for DPA are shown in Fig. 9e and the values and references are given in Supplementary Table S2. There are two inequivalent molecules in the DPA crystal unit cell ${ }^{69}$ and therefore two possible triplet-pair orientations, one parallel and one oblique. The oblique orientation results in spin statistical factors within the experimentally reported range.

In rubrene, the HL-RISC channel can contribute due to the favourable energy level alignment between $2 \times \mathrm{T}_{1}$, $\mathrm{T}_{2}$ and $\mathrm{S}_{1}$, which raises the value of $\eta$ close to the $\sim 60 \%$ measured for rubrene in solution ${ }^{18,21}$, indicated (with the reported experimental errors) in Fig. 9e. In solid rubrene, $\eta$ has been reported to reach $72 \%{ }^{65}$, also shown in Fig. 9e. Again, our model can explain this value through a combination of parallel molecular geometry, weakly exchangecoupled triplet-pairs and a partially active HL-RISC channel. The effectiveness of the HL-RISC channel is highly sensitive to the relative energy levels due to the exponential nature of the energy gap law and Boltzmann factors. Fig. 9d shows that variations on the order of $k_{B} T$ can have a large impact on $\eta$ and as shown in Supplementary Table S3, there is a considerable spread in the reported $\mathrm{T}_{2}$ energy level of rubrene. Finally, we note that in the absence of HL-RISC, $\eta$ increases only weakly as $\mathrm{T}_{2}$ is raised above ${ }^{3}$ (TT) and never reaches $100 \%$ as has been suggested ${ }^{14}$. In the presence of HL-RISC however, $\eta=100 \%$ is attained when $\mathrm{T}_{2}$ and ${ }^{3}$ (TT) are very close in energy and regardless of inter-molecular orientation.

\section{Conclusions}

In this work we have shown how factors rarely considered in discussions the of TTA-UC spin statistics can have a profound effect on the efficiency. In particular, we have explained why the oft repeated statement that TTA produces pure singlet, triplet and quintet encounter complexes in a 1:3:5 ratio contains an implicit assumption that the tripletpair states are strongly exchange-coupled. This is incompatible with experimentally measured magnetic field effects that can be explained only through weakly exchange-coupled triplet-pair states. When the triplet-pairs are weakly exchange-coupled, our simulations show that varying the inter-molecular orientation tunes the spin statistical factor from $\frac{2}{3}$ for parallel chromophores to $\frac{2}{5}$ for perpendicular chromophores, through variations in the spin mixing of the triplet-pair wavefunctions. We suggest that the origin of the commonly observed $40 \%$ value for acceptors such as DPA $^{8,9,28,40,70-74}$ is therefore considerably more subtle than has been assumed to date.

Our updated framework for calculating the spin statistical factor can also explain the higher values that have been measured for rubrene. Using transient absorption and pump-push-probe spectroscopy, we provided additional evidence for the recently reported high-level reverse intersystem crossing channel from $T_{2}$ to $S_{1}$ in rubrene. Based on the energy levels of $T_{1}, T_{2}$ and $S_{1}$, we modelled the effect of this channel and found that measured spin statistical factors of $60 \%$ for solution ${ }^{18,21}$ and $72 \%$ in the solid state ${ }^{65}$ can be readily understood in terms of chromophore orientation and high-level reverse intersystem crossing. 
This work points the way towards strategies for exceeding the spin statistical limit of TTA-UC. Control of intermolecular distance and geometry within the triplet-pair complexes can result in values up to $\frac{2}{3}$. Even better, harnessing high-level reverse intersystem crossing can make such considerations redundant, potentially allowing the spin statistical factor to reach unity.

\section{Experimental}

\section{Preparation of rubrene nanoparticles dispersed in PVA films}

Rubrene, purified by sublimation, was purchased from TCI and used as received. Poly(vinyl alcohol) (PVA, 99+\% hydrolyzed, average $M_{w}$ 130,000) was purchased from Merck and used as received. Films of rubrene nanoparticles (NPs) dispersed in PVA were prepared following previously reported procedures ${ }^{16,47}$. Briefly, a tetrahydrofuran solution of rubrene $(5 \mathrm{mM}, 3 \mathrm{~mL})$ was injected into an aqueous solution of sodium dodecyl sulfate $(10 \mathrm{mM}, 15 \mathrm{~mL})$. The NPs formed were collected by centrifugation and dispersed into an aqueous solution of PVA ( $8 \mathrm{wt} \%)$. The solution was cast onto quartz-coated glass substrates and dried overnight to form films. Prepared films were transferred to a nitrogen-filled glovebox and encapsulated using a glass coverslip and epoxy resin.

\section{Preparation of thermally evaporated rubrene films}

Rubrene was purchased from Ossila and used as received. Thin films were deposited on pre-cleaned quartz-coated glass substrates by thermal evaporation. The pressure during deposition was $2 \times 10^{-6}$ mbar or lower, the deposition rate was $0.3 \AA \mathrm{s}^{-1}$, the source temperature was $174-177^{\circ} \mathrm{C}$ and the final thickness was $125 \mathrm{~nm}$. The fresh, thermally evaporated films appeared smooth and featureless. The films were subsequently annealed on a hotplate at $185^{\circ} \mathrm{C}$ for $17 \mathrm{~min}$, resulting in visible crystallisation. The polycrystalline films were encapsulated using a glass coverslip and epoxy resin. All preparation was carried out inside a nitrogen-filled glovebox.

\section{Steady-state absorption and time-resolved photoluminescence spectroscopy}

Ground state absorption spectra were recorded with a UV-vis spectrophotometer (Cary60, Agilent). A Ti:sapphire regenerative amplifier (Solstice, Spectra-Physics) providing $800 \mathrm{~nm}$ pulses ( $90 \mathrm{fs}$ FWHM, $1 \mathrm{kHz}, 4 \mathrm{~mJ}$ ) was used to generate the pump beam for photoluminescence measurements. A portion of the $800 \mathrm{~nm}$ beam was frequency doubled in a BBO crystal to generate $400 \mathrm{~nm}$ pump pulses and focussed onto the sample. The photoluminescence was detected in reflection geometry by a spectrograph (Shamrock 303i, Andor) and a time-gated intensified charge-coupled device (iCCD; iStar DH334T-18U-73, Andor). A $435 \mathrm{~nm}$ long pass filter was used to eliminate pump scatter. Magnetic fields were applied transverse to the excitation beam using an electromagnet. Magnetic field strength was measured using a transverse Hall probe. Data processing procedures and further details regarding the TRPL setup have been reported previously ${ }^{26}$. The pump beam spot size was measured at the sample position by translating a razor blade through the focus and monitoring the transmitted power.

\section{Picosecond transient absorption spectroscopy}

A Ti:sapphire regenerative amplifier (Spitfire ACE PA-40, Spectra-Physics) providing $800 \mathrm{~nm}$ pulses (40 fs full-width at half-maximum (FWHM), $10 \mathrm{kHz}, 1.2 \mathrm{~mJ}$ ) was used to generate both the pump and probe beams. Tunable narrowband pump pulses at $532 \mathrm{~nm}$ were generated in an optical parametric amplifier (TOPAS Prime, Light Conversion). The pump was modulated by an optical chopper. Probe pulses spanning the range $350-750 \mathrm{~nm}$ and $830-1200 \mathrm{~nm}$ were generated by focusing a portion of the $800 \mathrm{~nm}$ beam through a continuously translating calcium fluoride or sapphire crystal respectively. Pump-probe delay was controlled using a motorized linear stage. Detection was carried out using a commercial instrument (Helios, Ultrafast Systems). The pump and probe polarizations were set to the magic angle. The pump beam spot size was measured at the sample position using a CCD beam profiler (Thorlabs). Transient absorption (TA) spectroscopy data were processed by background subtraction and chirp correction. 


\section{Pump-push-probe spectroscopy}

A Ti:sapphire regenerative amplifier (Solstice, Spectra-Physics) providing $800 \mathrm{~nm}$ pulses ( $90 \mathrm{fs}$ FWHM, $1 \mathrm{kHz}, 4 \mathrm{~mJ}$ ) was used to generate the pump, push and probe beams. Probe pulses spanning the range $460-700 \mathrm{~nm}$ were generated by focusing a portion of the $800 \mathrm{~nm}$ beam through a sapphire crystal. A second portion of the $800 \mathrm{~nm}$ beam was sent through an optical delay stage, followed by an 80:20 beamsplitter, and used to generate pump and push pulses. The $80 \%$ portion was passed through a BBO crystal, shortpass filter (Schott, BG39) and optical chopper to generate pump pulses ( $400 \mathrm{~nm}, 500 \mathrm{~Hz}, 0.2 \mathrm{~mJ} \mathrm{~cm}^{-2}$ ). The remaining $20 \%$ was delayed by a fixed $1070 \mathrm{ps}$ with respect to the pump and used as push pulses ( $800 \mathrm{~nm}, 1 \mathrm{kHz}, 1.2 \mathrm{~mJ} \mathrm{~cm}^{-2}$ ). The pump/push and probe polarizations were set to the magic angle and the three beams were overlapped at the sample adjacent to a reference beam obtained by passing the probe through a 50:50 beamsplitter. The reference is used to correct for shot-to-shot variation in the probe spectrum. The probe and reference beams were dispersed by a volume phase holographic grating (Wasastch) and detected by a pair of linear image sensors (S7030, Hamamatsu) driven and read out at the full laser repetition rate by a custom-built board from Entwicklungsbüro Stresing. TA data was acquired using home-built software. The pump and push beam spot sizes were measured at the sample position using a CCD beam profiler (Thorlabs).

\section{Author contributions}

DGB and JC conceived the project, which was supervised by JC, NK and NY. YS prepared samples. DGB performed the experiments, analysed the data and performed the simulations. DC assisted with the TA setup. DGB wrote the manuscript with input from JC, NY and YS.

\section{Conflicts of interest}

There are no conflicts to declare.

\section{Acknowledgements}

DGB thanks the EPSRC Centre for Doctoral Training in New and Sustainable Photovoltaics (EP/L01551X/1) for studentship support. YS thanks the JSPS Overseas Challenge Program for Young Researchers. JC thanks EPSRC for funding (EP/S002103/1 and EP/M025330/1). We thank EPSRC for a Capital Equipment award (EP/L022613/1 and EP/R042802/1), which provided the Lord Porter Laser Laboratory Facility used in this study. This work was partly supported by JSPS KAKENHI (grant numbers JP20H02713, JP20K21211, JP20H05676, JP18J21140).

\section{References}

[1] J. C. Goldschmidt and S. Fischer, Adv. Opt. Mater., 2015, 3, 510-535.

[2] J. Day, S. Senthilarasu and T. K. Mallick, Renew. Energy, 2019, 132, 186-205.

[3] J. Zhou, Q. Liu, W. Feng, Y. Sun and F. Li, Chem. Rev., 2015, 115, 395-465.

[4] L. Huang, E. Kakadiaris, T. Vaneckova, K. Huang, M. Vaculovicova and G. Han, Biomaterials, 2019, 201, 77-86.

[5] W. Wang, Q. Liu, C. Zhan, A. Barhoumi, T. Yang, R. G. Wylie, P. A. Armstrong and D. S. Kohane, Nano Lett., $2015,15,6332-6338$.

[6] Y. Sasaki, M. Oshikawa, P. Bharmoria, H. Kouno, A. Hayashi-Takagi, M. Sato, I. Ajioka, N. Yanai and N. Kimizuka, Angew. Chemie Int. Ed., 2019, 58, 17827-17833.

[7] L. Frazer, J. K. Gallaher and T. W. Schmidt, ACS Energy Lett., 2017, 2, 1346-1354. 
[8] V. Gray, D. Dzebo, M. Abrahamsson, B. Albinsson and K. Moth-Poulsen, Phys. Chem. Chem. Phys., 2014, 16, 10345-10352.

[9] V. Gray, K. Moth-Poulsen, B. Albinsson and M. Abrahamsson, Coord. Chem. Rev., 2018, 362, 54-71.

[10] M. J. Y. Tayebjee, D. R. McCamey and T. W. Schmidt, J. Phys. Chem. Lett., 2015, 6, 2367-2378.

[11] T. N. Singh-Rachford and F. N. Castellano, Coord. Chem. Rev., 2010, 254, 2560-2573.

[12] P. Bharmoria, H. Bildirir and K. Moth-Poulsen, Chem. Soc. Rev., 2020, 49, 6529-6554.

[13] T. N. Singh-Rachford and F. N. Castellano, J. Phys. Chem. A, 2008, 112, 3550-3556.

[14] S. Hoseinkhani, R. Tubino, F. Meinardi and A. Monguzzi, Phys. Chem. Chem. Phys., 2015, 17, 4020-4024.

[15] H. Kouno, Y. Sasaki, N. Yanai and N. Kimizuka, Chem. - A Eur. J., 2019, 25, 6124-6130.

[16] M. Kinoshita, Y. Sasaki, S. Amemori, N. Harada, Z. Hu, Z. Liu, L. K. Ono, Y. Qi, N. Yanai and N. Kimizuka, ChemPhotoChem, 2020, 4, 5271-5278.

[17] E. Radiunas, S. Raišys, S. Juršènas, A. Jozeliūnaitè, T. Javorskis, U. Šinkevičiūtė, E. Orentas and K. Kazlauskas, J. Mater. Chem. C, 2020, 8, 5525-5534.

[18] Y. Y. Cheng, B. Fückel, T. Khoury, R. G. C. R. Clady, M. J. Y. Tayebjee, N. J. Ekins-Daukes, M. J. Crossley and T. W. Schmidt, J. Phys. Chem. Lett., 2010, 1, 1795-1799.

[19] J. Zhao, S. Ji and H. Guo, RSC Adv., 2011, 1, 937-950.

[20] T. F. Schulze and T. W. Schmidt, Energy Environ. Sci., 2015, 8, 103-125.

[21] Y. Y. Cheng, T. Khoury, R. G. C. R. Clady, M. J. Y. Tayebjee, N. J. Ekins-Daukes, M. J. Crossley and T. W. Schmidt, Phys. Chem. Chem. Phys., 2010, 12, 66-71.

[22] B. Dick and B. Nickel, Chem. Phys., 1983, 78, 1-16.

[23] R. C. Johnson and R. E. Merrifield, Phys. Rev. B, 1970, 1, 896-902.

[24] K. Miyata, F. S. Conrad-Burton, F. L. Geyer and X.-Y. Zhu, Chem. Rev., 2019, 119, 4261-4292.

[25] A. J. Musser and J. Clark, Annu. Rev. Phys. Chem., 2019, 70, 323-351.

[26] D. G. Bossanyi, M. Matthiesen, S. Wang, J. A. Smith, R. C. Kilbride, J. D. Shipp, D. Chekulaev, E. Holland, J. E. Anthony, J. Zaumseil, A. J. Musser and J. Clark, Nat. Chem., 2021, 13, 163-171.

[27] X. Tang, R. Pan, X. Zhao, H. Zhu and Z. Xiong, J. Phys. Chem. Lett., 2020, 11, 2804-2811.

[28] R. Ieuji, K. Goushi and C. Adachi, Nat. Commun., 2019, 10, 5283.

[29] P. C. Tapping and D. M. Huang, J. Phys. Chem. C, 2016, 120, 25151-25157.

[30] S. L. Bayliss, A. D. Chepelianskii, A. Sepe, B. J. Walker, B. Ehrler, M. J. Bruzek, J. E. Anthony and N. C. Greenham, Phys. Rev. Lett., 2014, 112, 238701.

[31] R. Wang, C. Zhang, B. Zhang, Y. Liu, X. Wang and M. Xiao, Nat. Commun., 2015, 6, 8602.

[32] G. B. Piland, J. J. Burdett, D. Kurunthu and C. J. Bardeen, J. Phys. Chem. C, 2013, 117, 1224-1236.

[33] M. J. Y. Tayebjee, S. N. Sanders, E. Kumarasamy, L. M. Campos, M. Y. Sfeir and D. R. McCamey, Nat. Phys., 2017, $13,182-188$. 
[34] L. R. Weiss, S. L. Bayliss, F. Kraffert, K. J. Thorley, J. E. Anthony, R. Bittl, R. H. Friend, A. Rao, N. C. Greenham and J. Behrends, Nat. Phys., 2017, 13, 176-181.

[35] S. L. Bayliss, L. R. Weiss, A. Rao, R. H. Friend, A. D. Chepelianskii and N. C. Greenham, Phys. Rev. B, 2016, 94, 045204.

[36] S. L. Bayliss, L. R. Weiss, A. Mitioglu, K. Galkowski, Z. Yang, K. Yunusova, A. Surrente, K. J. Thorley, J. Behrends, R. Bittl, J. E. Anthony, A. Rao, R. H. Friend, P. Plochocka, P. C. M. Christianen, N. C. Greenham and A. D. Chepelianskii, Proc. Natl. Acad. Sci., 2018, 115, 5077-5082.

[37] T. Yago, K. Ishikawa, R. Katoh and M. Wakasa, J. Phys. Chem. C, 2016, 120, 27858-27870.

[38] J. J. Burdett, G. B. Piland and C. J. Bardeen, Chem. Phys. Lett., 2013, 585, 1-10.

[39] J. Mezyk, R. Tubino, A. Monguzzi, A. Mech and F. Meinardi, Phys. Rev. Lett., 2009, $102,087404$.

[40] T. W. Schmidt and F. N. Castellano, J. Phys. Chem. Lett., 2014, 5, 4062-4072.

[41] S. Reindl and A. Penzkofer, Chem. Phys., 1996, 211, 431-439.

[42] Y. Xu, X. Liang, X. Zhou, P. Yuan, J. Zhou, C. Wang, B. Li, D. Hu, X. Qiao, X. Jiang, L. Liu, S.-J. Su, D. Ma and Y. Ma, Adv. Mater., 2019, 31, 1807388.

[43] L. Ma, K. Zhang, C. Kloc, H. Sun, M. E. Michel-Beyerle and G. G. Gurzadyan, Phys. Chem. Chem. Phys., 2012, 14, 8307-8312.

[44] I. Breen, R. Tempelaar, L. A. Bizimana, B. Kloss, D. R. Reichman and D. B. Turner, J. Am. Chem. Soc., 2017, 139, 11745-11751.

[45] K. Miyata, Y. Kurashige, K. Watanabe, T. Sugimoto, S. Takahashi, S. Tanaka, J. Takeya, T. Yanai and Y. Matsumoto, Nat. Chem., 2017, 9, 983-989.

[46] I. Biaggio and P. Irkhin, Appl. Phys. Lett., 2013, 103, 263301.

[47] S. Amemori, Y. Sasaki, N. Yanai and N. Kimizuka, J. Am. Chem. Soc., 2016, 138, 8702-8705.

[48] R. C. Johnson, R. E. Merrifield, P. Avakian and R. B. Flippen, Phys. Rev. Lett., 1967, 19, $285-287$.

[49] L. Yarmus, J. Rosenthal and M. Chopp, Chem. Phys. Lett., 1972, 16, 477-481.

[50] C. Gao, S. K. K. Prasad, B. Zhang, M. Dvořák, M. J. Y. Tayebjee, D. R. McCamey, T. W. Schmidt, T. A. Smith and W. W. H. Wong, J. Phys. Chem. C, 2019, 123, 20181-20187.

[51] E. M. Gholizadeh, S. K. K. Prasad, Z. L. Teh, T. Ishwara, S. Norman, A. J. Petty, J. H. Cole, S. Cheong, R. D. Tilley, J. E. Anthony, S. Huang and T. W. Schmidt, Nat. Photonics, 2020, 14, 585-590.

[52] W. G. Herkstroeter and P. B. Merkel, J. Photochem., 1981, 16, 331-342.

[53] C. K. Yong, A. J. Musser, S. L. Bayliss, S. Lukman, H. Tamura, O. Bubnova, R. K. Hallani, A. Meneau, R. Resel, M. Maruyama, S. Hotta, L. M. Herz, D. Beljonne, J. E. Anthony, J. Clark and H. Sirringhaus, Nat. Commun., 2017, 8, 15953.

[54] F. Lewitzka and H.-G. Löhmannsröben, Zeitschrift für Phys. Chemie, 1986, 150, 69-86.

[55] L. Song and M. Fayer, J. Lumin., 1991, 50, 75-81.

[56] T. Zhu, Y. Wan, Z. Guo, J. Johnson and L. Huang, Adv. Mater., 2016, 28, 7539-7547.

[57] D. M. Finton, E. A. Wolf, V. S. Zoutenbier, K. A. Ward and I. Biaggio, AIP Adv., 2019, 9, 95027. 
[58] N. Aggarwal and A. Patnaik, J. Phys. Chem. B, 2017, 121, 3190-3201.

[59] N. Nijegorodov, V. Ramachandran and D. P. Winkoun, Spectrochim. Acta Part A Mol. Biomol. Spectrosc., 1997, 53, 1813-1824.

[60] F. Wang, G. Dukovic, L. E. Brus and T. F. Heinz, Phys. Rev. Lett., 2004, 92, 177401.

[61] A. Köhler and H. Bässler, Mater. Sci. Eng. R Reports, 2009, 66, 71-109.

[62] F. C. Spano, Acc. Chem. Res., 2010, 43, 429-439.

[63] C. D. Cruz, H. H. Choi, V. Podzorov, E. L. Chronister and C. J. Bardeen, J. Phys. Chem. C, 2018, 122, 1763217642.

[64] J. C. Beimborn, W. Zagorec-Marks and J. M. Weber, J. Phys. Chem. Lett., 2020, 11, 7212-7217.

[65] D. Y. Kondakov, T. D. Pawlik, T. K. Hatwar and J. P. Spindler, J. Appl. Phys., 2009, 106, 124510.

[66] W. Liu, S. Ying, R. Guo, X. Qiao, P. Leng, Q. Zhang, Y. Wang, D. Ma and L. Wang, J. Mater. Chem. C, 2019, 7, 1014-1021.

[67] R. A. Lampert and D. Phillips, J. Chem. Soc. Faraday Trans. 2 Mol. Chem. Phys., 1985, 81, 383-393.

[68] J. Gibson, A. P. Monkman and T. J. Penfold, ChemPhysChem, 2016, 17, 2956-2961.

[69] J. M. Adams and S. Ramdas, Acta Crystallogr. Sect. B, 1979, 35, 679-683.

[70] A. Monguzzi, F. Bianchi, A. Bianchi, M. Mauri, R. Simonutti, R. Ruffo, R. Tubino and F. Meinardi, Adv. Energy Mater., 2013, 3, 680-686.

[71] R. S. Khnayzer, J. Blumhoff, J. A. Harrington, A. Haefele, F. Deng and F. N. Castellano, Chem. Commun., 2012, 48, 209-211.

[72] R. W. MacQueen, Y. Y. Cheng, A. N. Danos, K. Lips and T. W. Schmidt, RSC Adv., 2014, 4, 52749-52756.

[73] N. Yanai, K. Suzuki, T. Ogawa, Y. Sasaki, N. Harada and N. Kimizuka, J. Phys. Chem. A, 2019, 123, 10197-10203.

[74] A. Olesund, V. Gray, J. Mårtensson and B. Albinsson, J. Am. Chem. Soc., 2021, 143, 5745-5754.

[75] A. Monguzzi, R. Tubino, S. Hoseinkhani, M. Campione and F. Meinardi, Phys. Chem. Chem. Phys., 2012, 14, 4322.

[76] K. Bera, C. J. Douglas and R. R. Frontiera, J. Phys. Chem. Lett., 2017, 8, 5929-5934.

[77] C. J. Imperiale, P. B. Green, E. G. Miller, N. H. Damrauer and M. W. B. Wilson, J. Phys. Chem. Lett., 2019, 10, 7463-7469.

[78] P. Irkhin and I. Biaggio, Phys. Rev. Lett., 2011, 107, 017402. 